\title{
Increase in Carbamazepine Serum Levels in Patient Treated with Voriconazole
}

\section{Alberto Sánchez-Romero ${ }^{1 *}$, Ana Valladolid-Walsh', Rafael García-Delgado and Esperan- za López-Rubio ${ }^{3}$}

\author{
${ }^{1}$ Department of Clinical Pharmacology, Complejo Hospitalario Universitario de Albacete, Spain \\ ${ }^{2}$ Department of Pharmacy, Complejo Hospitalario Universitario de Albacete, Spain \\ ${ }^{3}$ Department of Nephrology, Complejo Hospitalario Universitario de Albacete, Spain
}

*Corresponding author: Alberto Sánchez-Romero, Department of Clinical Pharmacology, Complejo Hospitalario Universitario, Hermanos Falcó 37, 02006, Albacete, Spain, Tel: 34-967-597234, Fax: 34-967-243952, E-mail: asanchez@sescam.jccm.es

\begin{abstract}
The addition of voriconazole to chronic treatment with carbamazepine $400 \mathrm{mg}$ twice a day was associated with increased carbamazepine serum levels $(14.1 \mathrm{mg} / \mathrm{l})$ and serum concentration dose ratios. Three days after the voriconazole was started, this ratio doubled its value to 1. Carbamazepine concentration as well as serum concentration dose ratio gradually decreased after treatment with voriconazole was discontinued, suggesting a probable interaction between the two drugs, according to the drug interaction probability scale. Although no clinical toxicity appeared with carbamazepine, the interaction can be considered severe and potentially toxic: early detection, suspension of carbamazepine and dose adjustment prevented such harm in this case. The likely mechanism is that voriconazole decreases carbamazepine metabolism.

To our knowledge this is the first reported case of interaction between voriconazole, as precipitant drug, and carbamazepine, as object drug. The interaction is not referenced in clinical databases as published cases, but as a foreseeable interaction.

Publication of this first clinical case reinforces the need of using both drugs associated with caution.

\section{Keywords}

Carbamazepine, Voriconazole, Drug interaction, Therapeutic drug monitoring
\end{abstract}

\section{Introduction}

Azole antifungals cause serious drug interactions when associated with drugs metabolised via CYP450 [1]. Some of these drugs (fluconazole, itraconazole, ketoconazole or miconazole) inhibit the main isoenzyme responsible for the metabolism of carbamazepine (CYP3A4) increasing its serum concentrations with concurrent administration. Voriconazole is metabolized by liver isoenzymes CYP2C19, CYP3A4 and CYP2C9 [2]. It is expected that voriconazole decreases metabolism of CYP3A4 substrates, such as carbamazepine [3], and recommendations are to modify treatment.
On the other hand, carbamazepine is an antiepileptic drug that is metabolized in the liver by CYP3A4 isoenzymes and to a lesser degree by CYP2C 8 and CYP3A5. It produces enzyme induction that increases azole antifungal metabolism and decreases their serum concentrations and effectiveness. It is possible that carbamazepine also decreases voriconazole levels significantly, contraindicating their simultaneous administration. There is a published case of a homozygous ultrarapid voriconazole metabolizing patient with low voriconazole levels comedicated with carbamazepine [4].

We report a case in which the addition of voriconazole to chronic treatment with carbamazepine was associated with increased carbamazepine serum levels and serum concentration dose ratios (SCD ratios).

We have not found published reports on interactions between voriconazole, as precipitant drug, and carbamazepine, as object drug. This interaction is not referenced in clinical databases as published cases, but as a foreseeable interaction.

\section{Clinical Case}

A 20-year-old Caucasian woman diagnosed of tuberous sclerosis, symptomatic partial epilepsy with complex partial seizures and polycystic kidney disease with stage III chronic disease and hypertension received chronic treatment with oral enalapril 15 mg once daily, carbamazepine $400 \mathrm{mg}$ twice daily and ferrous (II) glycine-sulfate $567 \mathrm{mg}$ twice daily. She was hospitalized for ongoing symptoms suggestive of pyelonephritis with sepsis criteria. After extracting culture samples, intravenous (IV) ciprofloxacin $400 \mathrm{mg}$ twice daily was prescribed. When Candida glabrata was isolated in urine cultures, IV voriconazole was started (standard loading dose of $400 \mathrm{mg}$ twice daily continued by $200 \mathrm{mg}$ twice daily maintenance regimen). On day 6 urine cultures were negative and treatment with voriconazole was stopped. Complications of the infectious process included: renal cyst rupture with massive hematuria and anemia and impaired renal function (serum creatinine 2.2-3.3 mg/dl), with hypoalbuminemia $(3 \mathrm{mg} / \mathrm{dl})$ and hypoproteinemia $(5.1 \mathrm{mg} / \mathrm{dl})$. 


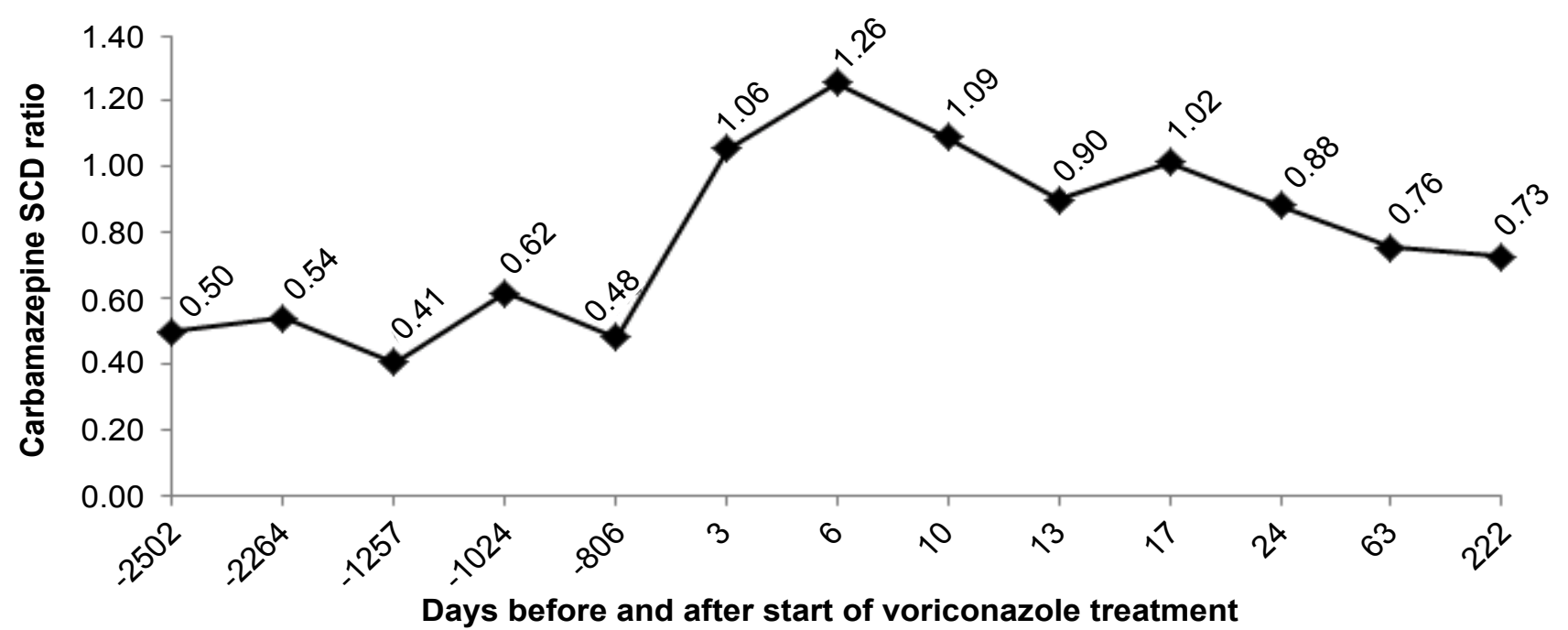

Figure 1: Time course of carbamazepine serum concentration-dose (SCD) ratio.

During admission other drugs prescribed included: omeprazole 20 mg twice daily, IV phytonadione $10 \mathrm{mg}$ twice daily for 2 days and paracetamol $1 \mathrm{~g}$ three times a day. The patient improved until hospital discharge.

\section{Carbamazepine serum concentration-dose relationship}

Figure 1 shows the time course of carbamazepine SCD ratio in this patient. During chronic treatment with carbamazepine at a dose of $400 \mathrm{mg}$ twice a day, therapeutic serum carbamazepine concentrations $(5.7-8.8 \mathrm{mg} / \mathrm{l})$ were reached. The SCD ratio ranged from 0.4 to 0.6 . During admission the patient received treatment with carbamazepine at the same dose and three days after starting treatment with voriconazole, supra therapeutic concentrations of carbamazepine were reached $(14.1 \mathrm{mg} / \mathrm{l}), \mathrm{SCD}$ ratio doubled its value to 1 . There was no clinical suspicion of toxicity caused by carbamazepine. Carbamazepine was then suspended for 24 hours and serum concentrations decreased to values similar to the previous $(8.2$ $\mathrm{mg} / \mathrm{l})$. The dose was then decreased to $200 \mathrm{mg}$ twice a day and SCD ratio reached its highest value 6 days after therapy with voriconazole was started.

Following discontinuation of treatment with voriconazole, the SCD ratio gradually decreased and was stable approximately one month later. It was necessary to increase the dose of carbamazepine to $600 \mathrm{mg}$ daily to maintain levels similar to the previous ones. The SCD ratio is calculated by dividing the serum concentration $(\mathrm{mg} / \mathrm{L})$ by the dose $(\mathrm{mg} / \mathrm{kg})$. The analytical technique used was fluorescence polarization immunoassay (Cobas Integra 400 Plus). Blood samples were drawn in the morning, 12 hours after the last dose of carbamazepine was administered.

\section{Discussion}

The alert sign of this possible interaction was the increase in serum levels and SCD ratios of carbamazepine after starting treatment with voriconazole.

Some of the most frequent side effects of carbamazepine are: a/ Neurologic: dizziness, drowsiness, ataxia, headache, diplopia, mydriasis, nystagmus, blurry vision and speech disorders; b/ Gastrointestinal: nausea and vomiting; c/ Cardiovascular: arrhythmias, cardiac conduction disorders.

No clinical toxicity appeared with carbamazepine despite the high serum concentration reached $(14.1 \mathrm{mg} / \mathrm{L})$, probably because of the early detection of this increase and immediate discontinuation of carbamazepine treatment for $24 \mathrm{~h}$ allowing serum concentration of carbamazepine to decrease to therapeutic values.

The event is consistent with the interactive properties of both drugs.
Several azole antifungals inhibit the main isoenzyme responsible for the metabolism of carbamazepine (CYP3A4). It is expected that voriconazole decreases metabolism of CYP3A4 substrates, such as carbamazepine, and recommendations are to modify treatment. But up to date there are no reports on interactions between voriconazole, as precipitant drug, and carbamazepine, as object drug.

The sequence or time course of the event is compatible with an interaction.

Three days after voriconazole was started, SCD ratio doubled its value and it peaked six days after. These short periods of time are explained by the administration of a loading dose and by the likely mechanism of interaction: enzyme inhibition may occur in 2-3 days, while enzyme induction requires more than 1 week.

SCD ratio of carbamazepine reached its maximum value concurring with the end of voriconazole administration. After this, it decreased progressively during one month, until stabilization. This slow decrease is explained by longer half-life of carbamazepine (10-20 h) that implies slower elimination.

Influence of other causes of the interaction is unlikely: it has been suggested that two associated drugs, omeprazole and ciprofloxacin, could reduce metabolism and increase bioavailability and serum concentration of carbamazepine [5,6]. These publications are singledose pharmacokinetic studies, we have found no published cases of interaction in patients chronically treated that describe auto induction of carbamazepine metabolism [7].

Treatment with ciprofloxacin and omeprazole started 11 and 6 days before, respectively, of the increase in carbamazepine concentrations and enzyme inhibition occurs at a time considerably lower (2-3 days), which coincides with treatment time with voriconazole before carbamazepine serum levels duplicated.

SCD ratio increase cannot be due to maturation of hepatic metabolic capacity, with slower clearance of carbamazepine, since the first serum level controls were performed during the adolescence period of the patient. Adults and adolescents show no significant differences in SCD ratios [8].

Analysis of this case according to the drug interactions probability scale (DIPS) showed a probable interaction corresponding to score of 5 [9].

Carbamazepine is eliminated by hepatic metabolism, mainly involving isoenzyme CYP3A4. Voriconazole inhibits "in vitro" activity of this isoenzyme [10,3]. There have been reports of increased serum carbamazepine concentrations after associating other azole antifungals with treatment [11]. 
This interaction is probably due to inhibition of biotransformation of carbamazepine, caused by carbamazepine-voriconazole competitive displacement from binding sites of CYP3A4. It can be considered severe: it doubled the concentration of carbamazepine and it was potentially toxic.

The existence of another mechanism of the interaction between these drugs contraindicates its use in patients with hepatic impairment. This interaction is twofold: voriconazole increases serum concentration of carbamazepine with potential toxicity, whereas carbamazepine decreases voriconazole concentration and its therapeutic effect.

\section{Conclusion}

Publication of this first clinical case of interaction between voriconazole, as precipitant drug, and carbamazepine, as object drug reinforces the need of using both drugs associated with caution and monitoring carbamazepine serum concentrations.

\section{References}

1. Andes D, Azie N, Yang H, Harrington R, Kelley C, et al. (2016) Drug-drug interaction associated with mold-active triazoles among hospitalized patients. Antimicrob Agents Chemother 60: 3398-3406

2. Geist MJ, Egerer G, Burhenne J, Riedel KD, Weiss J, et al. (2013) Steadystate pharmacokinetics and metabolism of voriconazole in patients. J Antimicrob Chemother 68: 2592-2599.
3. Nivoix Y, Levêque D, Herbrecht R, Koffel JC, Beretz L, et al. (2008) The enzymatic basis of drug-drug interactions with systemic triazole antifungals. Clin Pharmacokinet 47: 779-792.

4. Malingré MM, Godschalk PC, Klein SK (2012) A case report of voriconazole therapy failure in a homozygous ultrarapid CYP2C $19 * 17 / * 17$ patient comedicated with carbamazepine. Br J Clin Pharmacol 74: 205-206.

5. Dixit RK, Chawla AB, Kumar N, Garg SK (2001) Effect of omeprazole on the pharmacokinetics of sustained-release carbamazepine in healthy male volunteers. Methods Find Exp Clin Pharmacol 23: 37-39.

6. Shahzadi A, Javed I, Aslam B, Muhammad F, Asi MR, et al. (2011) Therapeutic effects of ciprofloxacin on the pharmacokinetics of carbamazepine in healthy adult male volunteers. Pak J Pharm Sci 24: 63-68.

7. Böttiger $Y$, Bertilsson L (1995) No effect on plasma carbamazepine concentration with concomitant omeprazole treatment. Drug Invest 9: 180-181.

8. Sánchez A, Durán JA, Serrano JS (1986) Steady-state carbamazepine plasma concentration-dose ratios in epileptic patients. Clin Pharmacokinet 11: 411-414

9. Horn JR, Hansten PD, Chan LN (2007) Proposal for a new tool to evaluate drug interaction cases. Ann Pharmacother 41: 674-680.

10. Jeu L, Piacenti FJ, Lyakhovetskiy AG, Fung HB (2003) Voriconazole. Clin Ther 25: 1321-1381.

11. Venkatakrishnan K, von Moltke LL, Greenblatt DJ (2000) Effects of the antifungal agents on oxidative drug metabolism: clinical relevance. Clin Pharmacokinet 38: 111-180. 